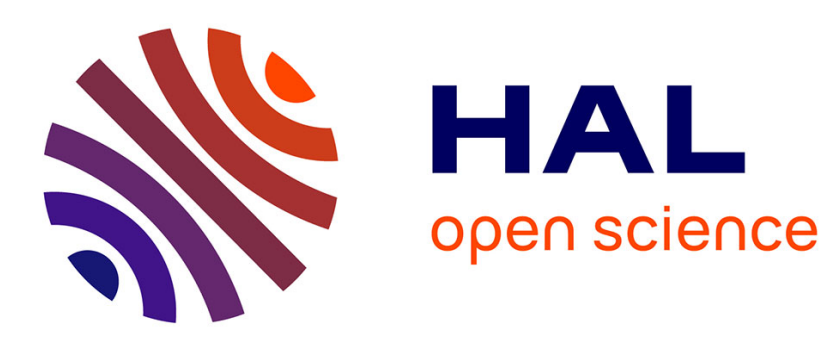

\title{
LANGEVIN APPROACH TO NUCLEAR DISSIPATIVE DYNAMICS
}

\author{
Y. Abe, C. Grégoire, H. Delagrange
}

\section{To cite this version:}

Y. Abe, C. Grégoire, H. Delagrange. LANGEVIN APPROACH TO NUCLEAR DISSIPATIVE DYNAMICS. Journal de Physique Colloques, 1986, 47 (C4), pp.C4-329-C4-338. 10.1051/jphyscol:1986436 . jpa-00225802

\section{HAL Id: jpa-00225802 https://hal.science/jpa-00225802}

Submitted on 1 Jan 1986

HAL is a multi-disciplinary open access archive for the deposit and dissemination of scientific research documents, whether they are published or not. The documents may come from teaching and research institutions in France or abroad, or from public or private research centers.
L'archive ouverte pluridisciplinaire HAL, est destinée au dépôt et à la diffusion de documents scientifiques de niveau recherche, publiés ou non, émanant des établissements d'enseignement et de recherche français ou étrangers, des laboratoires publics ou privés. 


\title{
LANGEVIN APPROACH TO NUCLEAR DISSIPATIVE DYNAMICS
}

\author{
Y. ABE, C. GRÉgoire* and H. DELAGRANGE* \\ Research Institute for Fundamental Physics, Kyoto University, \\ Kyoto 606, Japan \\ "Grand Accélérateur National d'Ions Lourds, BP 5027. \\ F-14021 Caen Cedex, France
}

\begin{abstract}
Resumé - La méthode de Langevin s'est presentée comme un cadre intuitif et phénoménologique pour descrire les phénomènes dissipatifs nucléeires tel que la reaction d!ions lourds et la désintegration fissionique. Nous présentons une méthode d'intégrer directement l'equation de Langevin avec la force de Langevin simulée par ordinateur: Quelques examples sont donnés en cas du mouvement libre de la particule Brownian et de la fission nucléaire comme une diffusion par-dessus la barrière.
\end{abstract}

\begin{abstract}
Langevin approach is proposed as an intuitive phenomenological framework to describe nuclear dissipative phenomena such as heavy ion reactions and fission decay. We present a method to integrate Langevin equation directly with the computer-simulated langevin force. Examples are given for a free motion of Brownian particle and for nuclear fission as a diffusion over a barrier.
\end{abstract}

\section{Introduction}

Deep inelastic collisions between heavy ions have shown dissipative phenomena, for example, dampings of the relative motion, and hence have driven nuclear physicists to interest themselves in non-equilibrium statistical theories. slow collective degrees of freedom with a heavy mass are looked as a Brownian particle and fast nucleon degrees of freedom as a heat bath. This picture relies upon the basic assumption that an equilibration time of nucleon degrees of freedom is much shorter than a typical time scale of collective motions, i.e., the time over which collective variables change significantly. Many efforts have been devoted to clarify a validity of the assumption and to derive physical parameters describing dissipative collective motions, for example friction and diffusion coefficients, from the microscopic Hamiltonians. This is out of the present talk and one can refer to extensive reviews $/ 1 /$. The subject of the present talk is concerning how to describe nuclear dissipative dynamics. So far we have employed the classical trajectory equation or Fokker-Planck equation. It is really bizarre that we have never utilized Langevin equation, which is more intuitive than Fokker-Planck one. Actually the former is more general than the latter one. As will be briefly discussed, it can describe non-Markovian process, which is likely in nuclear dissipative dynamics because collective masses are not so large as in the Brownian particle floating on fluia, compared with that of particles in the heat bath. The purpose of the present talk is to propose Langevin approach for a description of nuclear dissipative phenomena. In addition to its intuitiveness and its generality, it has also a practical advantage to accommodate many collective degrees of freedom straightforwardly. First we will remind the equivalence of Langevin and Fokker-Planck equations under certain conditions. Next we will develop the iteration riethod to solve stochastic equations directly with the computer simulated Langevin force. Finally we will discuss about results obtained by numerical calculations. 
2. Reminder of Langevin and Fokker-Planck Equations: Equivalence

In order to clarify the relation between Langevin and Fokker-Planck equation, we will derive Fokker-Planck equation starting from Langevin equation $/ 2 /$. Following Langevin, a Brownian particle floating on a fluid, i.e., a slow motion with heavy mass exerted frequently by random force is described by the equation,

$$
\frac{d}{d t} u=-\gamma \cdot u+\frac{1}{m} R(t)
$$

, where $u$ is a velocity of the Brownian particle with mass $m, \gamma$ is a friction coefficient, and $R(t)$ is a random force which is plausibly assumed to be Gaussian due to a large number of exertions, with the following properties;

$$
\begin{aligned}
& \text { (a) }\langle R(t)\rangle=0 \\
& \text { (b) }\left\langle R\left(t_{1}\right) R\left(t_{2}\right)\right\rangle=2 \pi I_{R} \cdot \delta\left(t_{1}-t_{2}\right) \text {. }
\end{aligned}
$$

The property (b) means no time-correlation among random forces and is essential to derive Fokker-Planck equation as will be seen in the following. In other words, Fokker-Planck equation applies only to Markovian processes.

We staxt with (stochastic) Lioville equation which describes the conservation of probability, i.e., with the continuity equation for probability,

$$
\frac{\partial}{\partial t} f(u, t)=-\frac{\partial}{\partial u}(\dot{u}(t) \cdot f(u, t))
$$

, where $\dot{u}(t)=\frac{d}{d t} u(t)$ and $f$ is a distribution function in velocity space. Inserting Eq. (2.1) into Eq.(2.2), we obtain

$$
\begin{aligned}
\frac{\partial}{\partial t} f(u, t) & =\Omega(u, t) \cdot f(u, t) \\
\Omega(u, t) & =\frac{\partial}{\partial u}\left(Y \cdot u-\frac{1}{m} R\right)
\end{aligned}
$$

Integrating Eq. (2.3) over $t \sim t+\Delta t$, where $\Delta t$ is much larger than the time scale of the random force $R(t)$ and still smallex than that of the macroscopic motion of the Brownian particle.

$$
\begin{aligned}
f(u, t+\Delta t) & =f(u, t)+\int_{t}^{t+\Delta t} d t_{1} \Omega\left(u, t_{1}\right) \cdot f\left(u, t_{1}\right) \\
& =\left[1+\int_{t}^{t+\Delta t} d t_{1} \Omega\left(u, t_{1}\right)+\int_{t}^{t+\Delta t} d t_{1} \int_{t}^{t_{1}} d t_{2} \Omega\left(u, t_{1}\right) \cdot \Omega\left(u, t_{2}\right)+\ldots . . . \cdot f(u, t)\right.
\end{aligned}
$$

Taking an average over all possible variations of $R(t)$ with the properties (a) and (b),

$$
\begin{aligned}
& \left\langle\Omega\left(u, t_{1}\right)\right\rangle=\frac{\partial}{\partial u} \gamma \cdot u, \\
& \left\langle\Omega\left(u, t_{1}\right) \cdot \Omega\left(u, t_{2}\right)\right\rangle=\frac{\partial}{\partial u} \gamma \cdot u \frac{\partial}{\partial u} \gamma \cdot u+\frac{1}{m^{2}} \frac{\partial^{2}}{\partial u^{2}} 2 \pi I_{R} \cdot \delta\left(t_{1}-t_{2}\right)
\end{aligned}
$$

where $I_{R}=m \cdot \gamma \cdot k T / \pi$ with Boltzmann constant $k$ and the temperature of heat bath $T$ from the fluctuation-dissipation theorem as will be simply discussed below. After integration, we take the limit $\Delta t \rightarrow 0$. We can readily see that the first term of the second equation and the higher order terms will vanish with the aid of the Gaussian assumption. Hence we have

$$
\frac{\partial}{\partial t} d(u, t)=\frac{\partial}{\partial u}\left(\gamma \cdot u+\frac{Y}{m} k T \frac{\partial}{\partial u}\right) \cdot d(u, t)
$$

, where a denotes a distribution function of slowly varying argument, whereas $f$ in Eq's(2.2) and (2.3) is that of rapidly varying stochastic variable. Eq. (2.4) is the diffusion equation in velocity space, i.e., Fokker-Planck equation. 
Here we note that Eq. (2.4) has a solution of the Boltzmann distribution as a stationary solution, i.e., the formula $I_{R}=\mathrm{m} \cdot \gamma \cdot \mathrm{kT} / \pi$ guarantees the correct thermal equilibration of the Brownian particle with the heat bath. This is seen more directly by calculating a mean kinetic energy $\frac{1}{2} \mathrm{~m}\left\langle\mathrm{u}^{2}\right\rangle$ with Langevin equation (2.1). A solution of Eq.(2.1) is

$$
u=u_{0} e^{-\gamma \cdot\left(t-t_{0}\right)}+\int_{t_{0}}^{t} d t^{\prime} e^{-\gamma \cdot\left(t-t^{\prime}\right)} \cdot \frac{1}{m} R\left(t^{\prime}\right) .
$$

Again using the property (b),

$$
\left\langle u^{2}\right\rangle=u_{0}^{2} \cdot e^{-2 \gamma \cdot\left(t-t_{0}\right)}+\frac{\pi \cdot I_{R}}{\gamma \cdot m^{2}}\left[1-e^{-2 \gamma \cdot\left(t-t_{0}\right)}\right] .
$$

Then

$$
\frac{1}{2} m<u^{2}>\rightarrow \frac{\pi}{2} \cdot \frac{1}{m \cdot \gamma} I_{R}=\frac{1}{2} \cdot k T, \text { as } t \rightarrow \infty .
$$

If we are interested in a motion of Brownian particle in an external force field, we start with a coupled Langevin equation,

$$
\begin{aligned}
& \frac{d}{d t} p=-\frac{\partial v}{\partial q}-\gamma \cdot p+R(t), \\
& \frac{d}{d t} q=\frac{1}{m} p,
\end{aligned}
$$

where $q$ and $p$ is a coordinate of Brownian particle and its conjugate momentum, and $v$ is a usual conservative potential. We can repeat the same procedure as in the case of the original Langevin equation, starting with Lioville equation in phase space and using the properties of random force (a) and (b). We will obtain Fokker-Planck equation in phase space,

$$
\frac{\partial}{\partial t} d(q, p, t)=\left\{-\frac{\partial}{\partial q} \frac{p}{m}+\frac{\partial}{\partial p} \frac{\partial v}{\partial q}+\frac{\partial}{\partial p}\left(\gamma p+m \gamma k T \frac{\partial}{\partial p}\right)\right\} d(q, p, t) .
$$

This is often called Kramers equation, whose stationary solutions were first discussed by Kramers /3/, and recently analyzed in detail to understand fission dynamics of highly excited compound nucleus by Weidenmuller, Grange, Gregoire and their collaborators $/ 4 /$.

Thus we have seen that Langevin equation is equivalent to Fokker-Planck equation under the properties of random force (a) and (b). In applications to nuclear dynamics, the former seems to be more intuitive than the latter. An average of Eq. (2.6) over all possible random forces gives us the classical trajectory equation, under the assumption that the potential does not change so much around the average,

$$
\begin{aligned}
\frac{\mathrm{d}}{\mathrm{dt}}\langle p\rangle & =-\frac{\partial v}{\partial\langle q\rangle}-\gamma \cdot\langle p\rangle, \\
\frac{\mathrm{d}}{\mathrm{d} t}\langle q\rangle & =\frac{1}{\mathrm{~m}}\langle p\rangle
\end{aligned}
$$

, which was extensively used to analyze heavy ion collisions by Bondorf, Gross and others /5/. It is worth noticing here that Eq. (2.7) is not enough to describe dissipative systems, because a friction force is always accompanied by Langevin force which gives rise to a fluctuation. This is generally true as long as the dissipation-fluctuation theorem holds. On the other hand, the coupled Langevin equation (2.6) properly describes not only the average trajectory but also the fluctuation, which sum up to be equivalent to the description by Fokker-planck equation.

Before proceeding to the problem of how to solve Langevin equation numerically, we should notice that we can easily generalize Langevin equation to non-Markovian 
processes by introducing a delayed friction,

$$
\frac{d}{d t} u=-\int d t^{\prime} \gamma\left(t-t^{\prime}\right) \cdot u\left(t^{\prime}\right)+\frac{1}{m} R(t) \text {. }
$$

Here the random force $R(t)$ can no more have the property (b) but has a timecorrelation given by $Y$,

$$
\left\langle R\left(t_{1}\right) \cdot R\left(t_{2}\right)\right\rangle=m \cdot k T \cdot \gamma\left(t_{1}-t_{2}\right)
$$

, which means that the random force does not have a white noise but a coloured one. This seems more suitable for nuclear physics and hence the generalized Langevin equation will have a wider applicability.

3. Direct Numerical Integrations of Langevin-Type Equations

3.1. The iteration method with simulated Langevin force

Langevin equation is so-called stochastic differential equation, which has rapidly changing force in addition to the ordinary one. As is well known, the former has no well-defined derivatives, and hence we cannot utilize usual methods of solving differential equations such as Runge-Kutta, Adams and so on, because they assume an existence of derivatives of the right-hand side of the differential equation. We will now try to solve the equation by the iteration method $/ 6 /$. We start with Langevin equation $(2.6)$ by rewriting as follows,

$$
\begin{aligned}
\frac{d}{d t} p & =h(p, q)+g \cdot \Gamma(t), \\
\frac{d}{d t} q & =\frac{1}{m} p,
\end{aligned}
$$

and

$$
\begin{aligned}
& h(p, q)=-\frac{\partial v}{\partial q}-\frac{\tilde{Y}}{m} p, \\
& g=\sqrt{\tilde{\gamma} \cdot k T}
\end{aligned}
$$

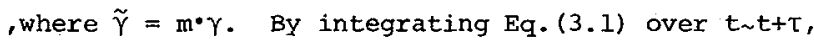

$$
\begin{aligned}
p(t+\tau)-p(t) & =\int_{t}^{t+\tau} a t^{\prime} h\left(p\left(t^{\prime}\right), q\left(t^{\prime}\right)\right)+g \int_{t}^{t+\tau} d t^{\prime} \Gamma\left(t^{\prime}\right) \\
& \cong \tau \cdot h(p(t), q(t))+g \cdot \tilde{\Gamma_{I}}(t), \\
q(t+\tau)-q(t) & =\frac{1}{m} \int_{t}^{t+\tau} d t^{\prime} p\left(t^{\prime}\right) \\
& \cong \tau \cdot \frac{p(t)}{m}
\end{aligned}
$$

Hence, starting at $t=0$ and iterating $n$ times, we will obtain $p(T)$ and $q(T)$ with $T=n \cdot \tau$. At each step of iterations, we need $\tilde{\Gamma}_{1}(t)=\int_{t}^{t+\tau} d t^{\prime} \Gamma\left(t^{\prime}\right)$, where random force $\Gamma(t)$
satisfies the conditions

$$
\begin{aligned}
& \text { (a) }\langle\Gamma(t)\rangle=0 \text {, } \\
& \text { (B) }\left\langle\Gamma\left(t_{1}\right) \cdot \Gamma\left(t_{2}\right)\right\rangle=2 \cdot \delta\left(t_{1}-t_{2}\right) \text {, }
\end{aligned}
$$

corresponding to the conditions $(a)$ and $(b)$ for $R(t)$. Since $\tilde{\Gamma}_{I}(t)$ is a sum of Gaussian random variable $\Gamma(t), \tilde{\Gamma}_{1}(t)$ is also Gaussian one and hence can be simulated by Gaussian random numbers which are generated, in a computer. From the property (B), 


$$
\begin{aligned}
& \left\langle\tilde{\Gamma}_{1}(t)^{2}\right\rangle=2 \tau \text {, hence we can write } \\
& \tilde{\Gamma}_{I}(t)=\int_{t}^{t+\tau} d t^{\prime} \Gamma\left(t^{\prime}\right)=\sqrt{\tau} \cdot \omega_{1}(t)
\end{aligned}
$$

, where $\omega_{1}(t)$ is a Gaussian random number with the properties

$$
\begin{aligned}
& \text { (a') }\left\langle\omega_{1}\right\rangle=0 \text {, } \\
& \text { (B') }\left\langle\omega_{1}^{2}\right\rangle=2 \text {. }
\end{aligned}
$$

By repeating the same procedures many times starting from the same initial conditions and averaging over all the realizations, we will obtain a solution of Langevin equation.

Eq's(3.4) and (3.5) are the first order approximation. If we want to improve an accuracy (in other words, if we want to use a larger step $\tau$ with good accuracy), we can expand the equations to higher order in $\tau$. For the sake of simplicity, we assume that $h(p, q)$ is of 1 st order in $p$ and of 2 nd order polynomials in $q$ separately, i.e., the potential is well described by the 3rd order polynomials in the coordinate q.

$$
\begin{aligned}
& h\left(p^{\prime}, q^{\prime}\right)=h(p, q)+\frac{\partial h}{\partial q}\left(p^{\prime}-p\right)+\frac{\partial h}{\partial q}\left(q^{\prime}-q\right)+\frac{1}{2} \frac{\partial^{2} h}{\partial q^{2}}\left(q^{\prime}-q\right)^{2} \\
& p^{\prime}=p+\left(p^{\prime}-p\right)
\end{aligned}
$$

, where $p^{\prime}$ and $q^{\prime}$ denote $p\left(t^{\prime}\right)$ and $q\left(t^{\prime}\right)$ respectively. Inserting the above expressions into the first lines of Eq's. (3.4) and (3.5), we obtain

$$
\begin{aligned}
p(t+\tau)-p(t)= & \tau \cdot h+\frac{1}{2} \tau^{2}\left(h q \frac{p}{m}+h p \cdot h\right) \\
& +\frac{1}{6} \tau^{3}\left\{h q q \cdot\left(\frac{p}{m}\right)^{2}+h p\left(h q \cdot \frac{p}{m}+h p \cdot h\right)+\frac{h q}{m} \cdot h\right\}+\ldots \\
& +g \cdot \tilde{\Gamma}_{1}(t)+h p \cdot g \cdot \tilde{\Gamma}_{2}(t)+\left(h p^{2}+\frac{h q}{m}\right) \cdot g \cdot \tilde{\Gamma}_{3}(t)+\ldots, \\
q(t+\tau)-q(t)= & \tau \cdot \frac{p}{m}+\frac{1}{2} \tau^{2} \frac{h}{m}+\frac{1}{6} \tau^{3} \cdot \frac{1}{m} \cdot\left(h q \frac{p}{m}+h p \cdot h\right)+\ldots \\
& +\frac{g}{m} \cdot \tilde{\Gamma}_{2}(t)+\frac{g}{m} \cdot h p \cdot \tilde{\Gamma}_{3}(t)+\ldots,
\end{aligned}
$$

, where $h p=\frac{\partial h}{\partial p}, h q=\frac{\partial h}{\partial q}$ and $h q q=\frac{\partial^{2} h}{\partial q^{2}}$.

$$
\begin{aligned}
& \tilde{\Gamma}_{2}(t)=\int_{0}^{\tau} d \tau^{\prime} \int_{t}^{t+\tau^{\prime}} d t^{\prime} \Gamma\left(t^{\prime}\right) \\
& \tilde{\Gamma}_{3}(t)=\int_{0}^{\tau} d \tau^{\prime} \int_{0}^{\tau^{\prime}} d \tau^{\prime \prime} \int_{t}^{t+\tau^{\prime \prime}} d t^{\prime} \Gamma\left(t^{\prime}\right) \\
& \ldots .
\end{aligned}
$$

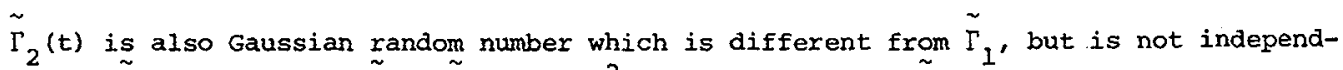
ent of $\tilde{\Gamma}_{1}(t)$, because $\left\langle\tilde{\Gamma}_{1}(t) \tilde{\Gamma}_{2}(t)\right\rangle=\tau^{2}$. We can simulate $\tilde{\Gamma}_{2}(t)$ as follows 


$$
\tilde{\Gamma}_{2}(t)=\tau^{3 / 2}\left[\frac{1}{2} \omega_{1}(t)+\frac{1}{2 \sqrt{3}} \omega_{2}(t)\right]
$$

so as to satisfy also $\left\langle\tilde{\Gamma}_{2}(t)^{2}\right\rangle=\frac{2}{3} \tau^{3}, \tilde{\Gamma}_{3}(t), \tilde{\Gamma}_{4}(t), \ldots$ will be generated in the same way. It is important to notice here that there appear no derivatives of the random force $\Gamma(t)$ in the above equations. Eq.'s(3.7) and (3.8) are the basic equations which are to be iterated to obtain solutions of Langevin equation (3.1). As is readily seen, if we want to include several collective degrees of freedom such as three dimensional relative motion between heavy ions, mass asymmetry in fission etc., we can simply add relevant variables into the set of the equations above. of course, we need more random numbers to describe their fluctuation, but we suffer no more complications.

In order to confirm a validity of the above iteration method, we can calculate an energy balance, $i . e .$, an enexgy gain due to kick-off by the random force and an energy loss due to the friction. In the equilibrium limit, they should compensate with each other. For the original Langevin equation, the energy variation of the Brownian particle over $t \sim t+\tau$ is

$$
\Delta E=\int_{t}^{t+\tau} d t^{\prime} F\left(t^{\prime}\right) \cdot u\left(t^{\prime}\right)=\frac{1}{m} \int_{t}^{t+\tau} d t^{\prime} F\left(t^{\prime}\right) \cdot p\left(t^{\prime}\right)
$$

, where $u\left(t^{\prime}\right)$ is the velocity and $F=F_{1}+F_{2}=-\frac{\tilde{Y}}{m} p+g \cdot \Gamma$. Inserting Eq. (3.7) into Eq. (3.11) and averaging over all possible realizations of the random force, we obtail

$$
\begin{aligned}
\langle\Delta E\rangle= & -\frac{\tilde{Y}}{m} \cdot \tau \cdot\left\{\frac{\left\langle p^{2}\right\rangle}{m}-k T\right\}+\left(\frac{\tilde{Y}}{m}\right)^{2} \cdot \tau^{2} \cdot\left\{\frac{\left\langle p^{2}\right\rangle}{m}-k T\right\} \\
& +2\left(\frac{\tilde{Y}}{m}\right)^{3} \cdot \tau^{3} \cdot\left\{\frac{\left\langle p^{2}\right\rangle}{m}-k T\right\}+\ldots .
\end{aligned}
$$

Thus $\langle\Delta \mathrm{E}\rangle=0$ at the equilibrium with the heat bath.

3.2. Comparison of results by the numerical integration with exact ones: original Langevin equation

As already given in Eq. (2.5), the analytic solution of Langevin equation (3.1) with $\mathrm{V}=0$ is simply given by

$$
\begin{aligned}
& p=p_{0} e^{\frac{\tilde{\gamma}_{m}}{m} t}+d t^{\prime} e^{-\frac{\tilde{\gamma}}{m}\left(t-t^{\prime}\right)} \cdot g \cdot \Gamma\left(t^{\prime}\right) \\
& q=q_{0}+\frac{p_{0}}{\tilde{\gamma}}\left[I-e^{-\frac{\tilde{\gamma}_{t}}{m}} 1+\frac{1}{\tilde{\gamma}} \int_{0}^{t} d t^{\prime}\left[1-e^{-\frac{\tilde{\gamma}}{m}\left(t-t^{\prime}\right)} l \cdot g \cdot \Gamma\left(t^{\prime}\right)\right.\right.
\end{aligned}
$$

, where $p_{0}$ and $q_{0}$ are the initial values respectively. Then

$$
\begin{aligned}
& \langle p\rangle=p_{0} e^{-\tilde{\gamma}_{t}}, \\
& \langle q\rangle=q_{0}+\frac{p_{0}}{\tilde{\gamma}}\left[1-e^{\left.-\frac{\tilde{Y}_{t}}{m}\right]},\right.
\end{aligned}
$$

And

$$
\left\langle\mathrm{p}^{2}\right\rangle=\mathrm{m} \cdot \mathrm{kT} \cdot\left[1-\mathrm{e}^{-2 \frac{\tilde{\gamma}}{\mathrm{m}} \cdot t}\right]+\mathrm{p}_{0}^{2} \mathrm{e}^{-2 \frac{\tilde{q}}{\mathrm{~m}} \cdot t},
$$




$$
\begin{aligned}
\left\langle\left(q-q_{0}\right)^{2}\right\rangle & =\left(p_{0}^{2}-3 m k T\right) / \tilde{\gamma}^{2}+2 k T / \tilde{\gamma} \cdot t+2 \cdot\left(2 m k T-p_{0}^{2}\right) / \tilde{\gamma}^{2} \cdot e^{-\frac{\tilde{Y}}{m} \cdot t} \\
& +\left(p_{0}^{2}-m k T\right) / \tilde{\gamma}^{2} \cdot e^{-2 \cdot \frac{\tilde{\gamma}}{m} \cdot t} .
\end{aligned}
$$

On the other hand, we will solve the equation numerically with the method explained above. We calculate $\mathrm{N}=10000$ examples by the use of Gaussian random numbers for $\left\{\omega_{1}(t)\right\}$ and average over them at each time step. The quality of the random numbers $\left\{\omega_{1}^{1}(t)\right\}$ is shown in Fig.1. In Fig.2, results obtained by the present method are

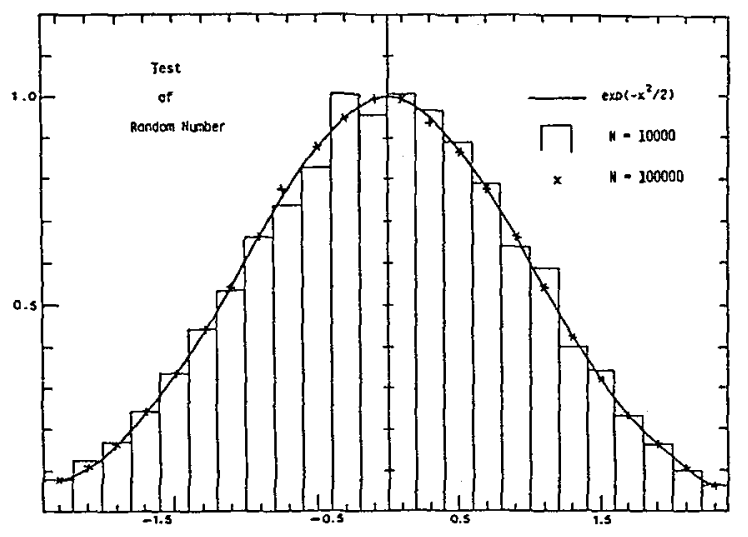

Fig. 1 - Example of Gaussian random numbers.

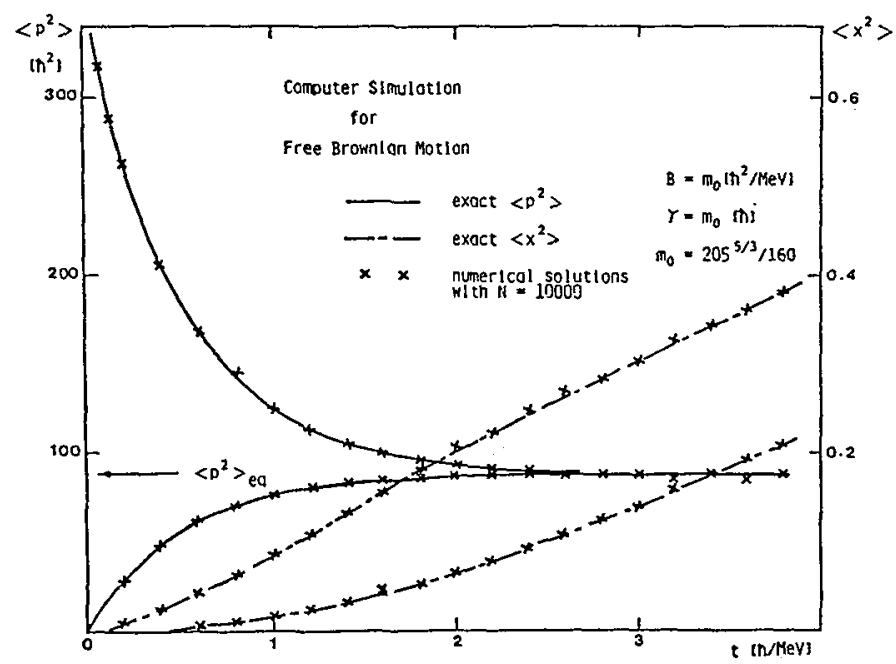

Fig. $2-\left\langle q^{2}\right\rangle$ and $\left\langle p^{2}\right\rangle$ of the free Brownian particle starting with two diffexent initial momenta $p_{0}$ 's. Mass and friction parmeters are the same as those in Fig. 3 . (see text) The present results $(x)$ are always very close to the exact values. 
compared with those of Eqs. (3.17) and (3.18) for the cases starting with $p_{0}=0$ and $\mathrm{p}_{0}=2 \cdot \mathrm{p}_{\mathrm{eq}}^{2}$ where $\mathrm{p}_{\text {eq }}^{2}=\mathrm{m} \cdot \mathrm{kT}$. The numerical results almost coincide with the analytic solution at any steps. We can see that the Brownian particle approaches to the thermal equilibrium with the heat bath, i.e., $\left\langle\mathrm{p}^{2}\right\rangle \rightarrow \mathrm{m} \cdot \mathrm{kT}=\mathrm{p}_{\mathrm{eq}}^{2}$. The errors are within 1 28. which is comparable with $I / \sqrt{\mathrm{N}}$. Of course $\langle\mathrm{p}\rangle$ and $\langle\mathrm{q}\rangle$ are much better reproduced, although not shown here.

\subsection{Calculations of fission decay width : escape over a barrier}

Next we take up a fission decay rate of highly excited compound nuclei as one of more realistic examples. Kramers viewed the nuclear fission decay as a diffusion process over a potential barrier, i.e., the collective elongation mode leading the nucleus to fission is assigned to a Brownian particle and non-collective nucleon degrees of freedom in the excited nucleus to the heat bath. This is not peculiar, because we know a lot of experimental data in heavy ion reactions which indicate a very fast thermalization of nucleon degrees of freedom, faster than some collective motions as discussed by previous speakers in this Conference.

We solve Eq. (3.1) which is equivalent to Kramers equation(2.7) with the present method starting with the same initial conditions as scheuter and Gregoire's/4/, i.e., the initial distribution is given by

$$
d(c, p)= \begin{cases}N \cdot \exp \left[-\left(p^{2} / 2 B+V(c)\right) / k T\right] & \text { for } c \leq c_{\text {saddle }}=1.8 \\ a\left(c=c_{\text {saddle }}\right) & \text { for } c>c_{\text {saddle }}\end{cases}
$$

, where $\mathrm{N}$ is a normalization constant. Physical parameters used are also the same as those employed by them. The collective mass of the elongation $B_{C}=m_{0}=A^{5 / 3 / 160 ~}$ $\left[\hbar^{2} / \mathrm{MeV}\right]$ and the friction coefficient $\tilde{\gamma}_{C}=f \cdot \tilde{Y}_{0}=m_{0}\left[h_{1}\right]$, where the mass number $A$ is 205 of At. The potential $\mathrm{V}$ is given as a function of the elongation coordinate $c$,

$$
V(c)= \begin{cases}37.46 \cdot(c-1)^{2} & \text { for } 0<c<1.27, \\ 8.0-18.73 \cdot(c-1.8)^{2} & \text { for } 1.27<c\end{cases}
$$

At each iteration step, we calculate a probability of the system being compound nucleus $P_{\text {C.N.' }}$ i.e., number of examples with $c<c_{\text {saddle }}$ devided by the total number of examples initially prepared, and then calculate escape rate as follows,

$$
r(t)=-\frac{1}{P_{C \cdot N}}-\frac{d P_{C \cdot N}}{d t}
$$

As the rate calculated at each step is still slightly fluctuating, we make a timeaveraging over $\Delta t$

$$
\begin{aligned}
& \langle r(t)\rangle_{\Delta t}=\frac{1}{\Delta t} \int_{t-\Delta t / 2}^{t+\Delta t / 2} d t \\
& =\frac{1}{\Delta t} \ln \left(P_{C . N .}(t-\Delta t / 2) / P_{C . N .}(t+\Delta t / 2)\right)
\end{aligned}
$$

Results are compared with those obtained by solving Fokker-Planck equation with the bundle propagation method/4/ whexe the momentum distribution is always assumed to be Gaussian and with the following Kramers formulae/3/, 


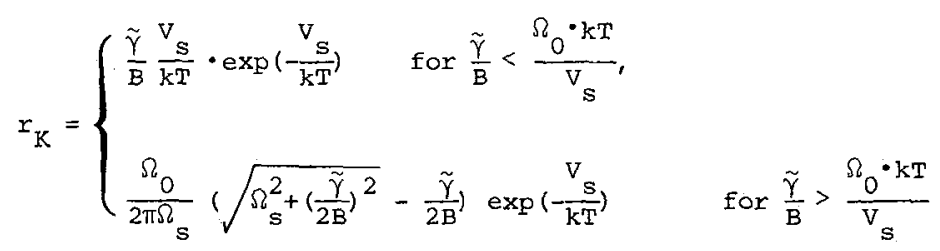

where $\Omega_{0}$ and $\Omega_{s}$ are the local frequencies in the minimum and at the sadale point, and $\mathrm{V}$ is the barcier height. Using the parameters given above, the critical friction coefficient is given as follows,

$$
\mathrm{f}_{\text {crit }}=\left(\frac{\tilde{\gamma}}{\gamma_{0}}\right)_{\text {crit }} \equiv \frac{\Omega_{0} \cdot \mathrm{kT}}{v_{s}}=0.325
$$

, with $\mathrm{kT}=\sqrt{\mathrm{E}^{*} / \mathrm{a}}$ where the excitation energy $\mathrm{E}^{*}$ is taken to be $80 \mathrm{MeV}$ and the level density parameter a to be $A / 10$.

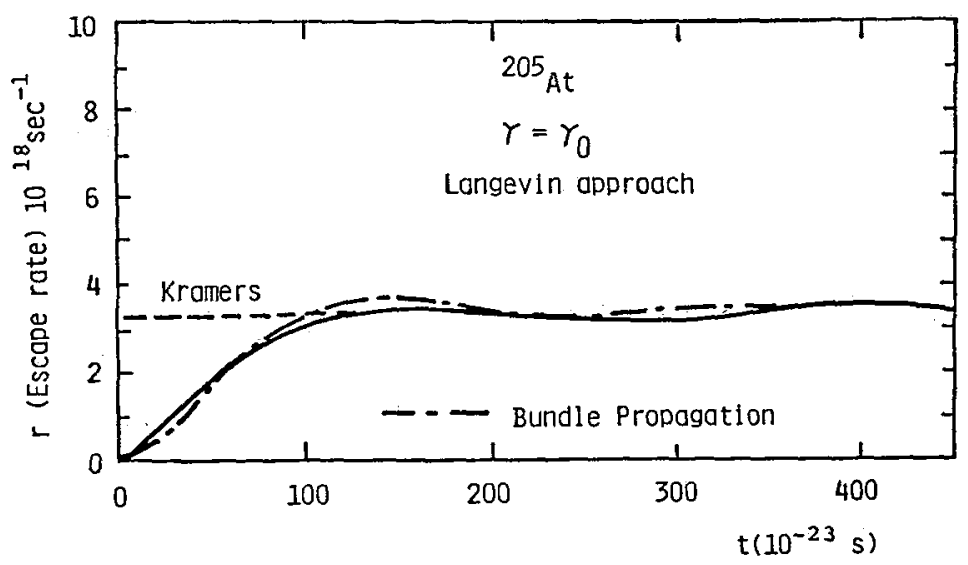

Fig. 3 - Time-dependence of calculated escape rate (soljg line) is compared with the bundle propagation and Kramers's limit, for fission of ${ }^{205} \mathrm{At}$.

In Fig.3, the escape rates are compared as functions of the time $t$. We can see that the present results are very similar to those by the bundle propagation method. Both show a transient behaviour at short time periods and then approach to the Kramers's limit given by the second formula of Eq. (3.21). It is rather surprising that the present calculation is so accurate, because the decay rate is not an average property of the total examples initially prepared, but is deternined by a tiny portion of them, although they amount to 10 . In order to confirm an applicability of Langevin approach further, we calculate the escape rate over a wide range of the friction coefficient. We compare stationary. values as functions of $f=\tilde{Y} / \tilde{Y}$ in $F i g .4$. The present calculations surprinsingly well reproduce Kramers's limit in both low and high friction regimes. The bundle propagation method is also very accurate in high friction regime, but fails in lower side. This is probably due to the fact that the assumption of the Gaussian distribution in momentum space does not persist to be a good approximation in low frictions, because there the system takes a long time to approach to the thermalization even in momentum space. The arrow in Fig. 4 points the critical value $f$ which is a border between the two regions. In the region around the arrow, howeverr, both Kramers's formulae are shown not to provide correct stationary limits. The present calculations well bridge Kramers's two limiting cases. 


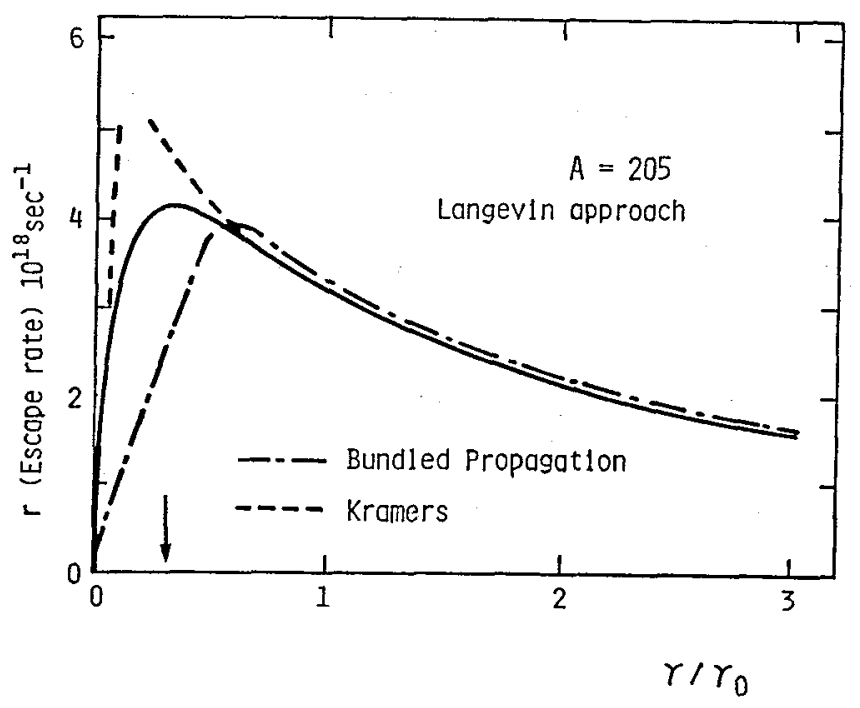

Fig. 4 - Dependence of calculated stationary escape rate on friction coefficient is compared with the bundle propagation method and two Kramers's limits. Present calculations well bridge two limits.

\section{Concluding Remarks}

As I mentioned in the introduction, it is really peculiar that most nuclear physicists prefer to use Fokker Planck equation rather than Langevin equation. The reason might be due to the fact that the former equation is a partial differen.. tial equation without any stochastic variables, which allows us to manipulate analytically as Kramers did. On the other hand, the latter one is an ordinary differential equation but with Langevin force, which is not easily manageable with usual analytical methoas. However, by virtue of its intuitiveness and its generality, it is worth developing some ways of solving Langevin equation and applying it to nuclear dissipative dynamics. In the present talk, we have developed the iteration method with the computer-simulated Langevin force, and have succeeded in solving the nuclear fission decay process as a diffusion over the barrier. An extension to include several degrees of freedom is straight-forward and hence we expect that the present method applies to heavy ion collisions leading to highly excited compound nuclei, which subsequently decay through fission-like processes, only if we can assume that the nucleon thermalization is much faster than collective motions.

One of the authors (Y.A.) acknowledges the GANIL laboratory for the kind hospitality extended to him during his stay in Caen, while most of the present works have been made. Numerical calculations were performed by the computers at GANIL and at the Data Processing Center of Kyoto University.

\section{References}

1/ Deubler, H.H., Dietrich, K, and Hofmann, H., Heavy Ion Collisions, vol.2, Chapt. 5, ed. by R. Bock, North-Holland Publishing Company (1980) .

Weidenmuller, H.A., Progress in Particle and Nuclear Physics, vol.3, p.49, ed. by D. Wilkinson, Pergamon, Oxford (1980).

/2/ Kubo, R., Reports in Progress in Physics, 29 (1966) 255.

/3/ Kramers, H.A., Physica VII, no.4 (I940) $28 \overrightarrow{4}$.

/4/ Grange, P., Li, J.Q. and weidenmuller, H.A., Phys. Rev. C27 (1983) 2063.

Scheuter, F. and Hofmann, H., Nucl. Phys. A394 (1983) 477.

Scheuter, F. and Grégoire, C., Proc. of XII Intern. Symp. on Nuclear Physics, ISSN0138-2950, p.102, ed. by R. Reif and J. Teichert, Gaussing, German Democratic Republic (1982).

/5/ Bondorf, J.P., Sobel, M.I. and Sperber, D., Phys. Rep. 15C (1974) 83. Gross, D.H.E. and Kalinowski, H., Phys. Letters 48B (1974) 302.

16/ Risken, H., The Fokker-Planck Equation, Chapt.3. Springer-Verlag, Berlin (1984). 